IRSTI 27.29.25

https://doi.org/10.26577/ijmph-2019-i2-2

\author{
${ }^{1}$ M. Akhmet, ${ }^{2}$ M. Tleubergenova, ${ }^{2}$ A. Zhamanshin \\ ${ }^{1}$ Middle East Technical University, 06800 Ankara, Turkey, \\ e-mail: marat@metu.edu.tr, \\ ${ }^{2}$ Aktobe Regional State University, Aktobe, Kazakhstan, \\ e-mail: madina_1970@mail.ru, akylbek78@mail.ru
}

\title{
Strongly unpredictable solutions of difference equations
}

\begin{abstract}
It so happens that the line of oscillations in the classical theory of dynamical systems, which is founded by H.Poincar'e and G.Birkhoff was broken at Poisson stable motions. The next oscillations were considered as actors of chaotic processes. This article discusses the new type of oscillations, unpredictable sequences, the presence of which proves the existence of Poincare chaos. The sequence is defined as an unpredictable function on the set of integers. The results continue the description of chaos which isinitiated from a single motion, an unpredictable one. To demonstrate the effectiveness of the approach, the existence and uniqueness of the unpredictable solution for a quasilinear difference equation are proved. An example with numerical simulations is presented to illustrate the theoretical results. Since unpredictability is request for all coordinates of solutions, the concept of strong unpredictability can be useful for investigation of neural networks, brain activity, robotics, where complexity is related to optimization and effectiveness.
\end{abstract}

Key words: Difference equations, Strongly unpredictable solutions, Existence and uniqueness, Asymptotical stability.

\section{Introduction}

Throughout the paper, $\mathbb{R}, \mathbb{N}$ and $\mathbb{Z}$ will stand for the set of real, natural and integer numbers, respectively. Additionally, $x=x_{i}, i \in \mathbb{Z}$, and the norm $\|x\|_{1}=\sup _{\mathbb{Z}}\left\|x_{i}\right\|, \quad$ where $\quad x_{i}=$ $\left(x_{i}^{1}, \ldots, x_{i}^{p}\right), x_{i}^{j} \in \mathbb{R},\left\|x_{i}\right\|=\max _{1 \leq j \leq p}\left|x_{i}^{j}\right|, j=$ $1,2, \ldots, p, p \in \mathbb{N}$, will be used. The following definition isone of the main in our study.

Definition 1. [1,2] A bounded sequence $\kappa_{i}, i \in \mathbb{Z}$, in $\mathbb{R}^{p}$ is called unpredictable if there exist a positive number $\varepsilon_{0}$ and sequences $\zeta_{n}, \eta_{n}, n \in \mathbb{N}$, of positive integers both of which diverge to infinity such that $\left\|\kappa_{i+\zeta_{n}}-\kappa_{i}\right\| \rightarrow 0$ as $n \rightarrow \infty$ for each $i$ in bounded intervals of integers and $\left\|\kappa_{\zeta_{n}+\eta_{n}}-\kappa_{\eta_{n}}\right\| \geq \varepsilon_{0}$ for each $n \in \mathbb{N}$.

Some coordinates of an unpredictable sequence may be not unpredictable. This is why, in the following definition, we consider a stronger version of the concept.

Definition 2. A bounded sequence $\kappa_{i}, i \in \mathbb{Z}$, in $\mathbb{R}^{p}$ is called strongly unpredictable if there exist a positive number $\varepsilon_{0}$ and sequences $\zeta_{n}, \eta_{n}, n \in \mathbb{N}$, of positive integers both of which diverge to infinity such that $\left\|\kappa_{i+\zeta_{n}}-\kappa_{i}\right\| \rightarrow 0$ as $n \rightarrow \infty$ for each $i$ in bounded intervals of integers and $\left|\kappa_{\zeta_{n}+\eta_{n}}^{j}-\kappa_{\eta_{n}}^{j}\right| \geq$ $\varepsilon_{0}$ for each $j=1, \ldots, p$ and $n \in \mathbb{N}$.

Inthispaper, a strongly unpredictable sequence and a strongly unpredictable solutionare understood as mentioned in Definition 2. We investigate the existence, uniqueness andstability of strongly unpredictable solutions of a non-linear difference equation.

The research of complex dynamics as well as differential equations with singularities has been of great interest in recent decades [3-8].

\section{Main result}

Let us consider the following discrete equation

$$
z_{i+1}=B z_{i}+h\left(z_{i}\right)+\psi_{i},(1)
$$

where $z_{i}=\left(z_{i}^{1}, \ldots, z_{i}^{p}\right), z_{i}^{j} \in \mathbb{R}, i \in \mathbb{Z}, j=$ $1, \ldots, p, B=\operatorname{diag}\left(b_{1}, b_{2}, \ldots, b_{p}\right)$ is a real valued nonsingular matrix, $h=\left(h_{1}, h_{2}, \ldots, h_{p}\right), h: \mathbb{R}^{p} \rightarrow$ $\mathbb{R}^{p}, p \in \mathbb{N}$, is a continuous function, and $\psi_{i}=$ $\left(\psi_{i}^{1}, \psi_{i}^{2}, \ldots, \psi_{i}^{p}\right), i \in \mathbb{Z}$, is a strongly unpredictable sequence.

Since $\psi_{i}, i \in \mathbb{Z}$, is a strongly unpredictable sequence, there exist a positive number $\varepsilon_{0}$ and 
Since $\psi_{i}, i \in \mathbb{Z}$, is a strongly unpredictable sequence, there exist a positive number $\varepsilon_{0}$ and sequences $\zeta_{n}, \eta_{n}, n \in \mathbb{N}$, of positive integers both of which diverge to infinity such that $\left\|\psi_{i+\zeta_{n}}-\psi_{i}\right\| \rightarrow$ 0 as $n \rightarrow \infty$ for each $i$ with $\alpha \leq i \leq \beta, \alpha, \beta \in \mathbb{Z}$, and $\left|\psi_{\zeta_{n}+\eta_{n}}^{j}-\psi_{\eta_{n}}^{j}\right| \geq \varepsilon_{0, j}=1, \ldots, p$ for each $n \in \mathbb{N}$. Denote $M_{\psi}=\sup _{\mathbb{R}}\left\|\psi_{i}\right\|$.

Let $U$ be the set of infinite sequences $x=$ $\left\{x_{i}\right\}, x_{i}=\left(x_{i}^{1}, \ldots, x_{i}^{p}\right), x_{i}^{j} \in \mathbb{R}, i \in \mathbb{Z}, j=$

\section{$1,2, \ldots, p$, such that:}

(A1) $\|x\|_{1}<H$, where $H$ is a positive real number;

(A2) There exists a sequences $\zeta_{n}, \zeta_{n} \rightarrow \infty$ as $n \rightarrow \infty$ such that $\left\|x_{i+\zeta_{n}}-x_{i}\right\| \rightarrow 0$ as $n \rightarrow \infty$ on each bounded interval of integers.

The following conditions are needed throughout this paper.

(C1) There exists a positive number $M_{h}$ such that $\sup _{\|x\|<H}\|h(x)\|<M_{h}$;

(C2) There exists a positive number $L_{h}$ such that $\|h(x)-h(y)\| \leq L_{h}\|x-y\| \quad$ for $\quad$ all $\quad\|x\|<$ $H,\|y\|<H$;

(C3) $\bar{b}+L_{h}<1$, where $\bar{b}=\max _{k}\left|b_{k}\right|$;

(C4) $\left(M_{h}+M_{\psi}\right) \frac{1}{1-\bar{b}}<H$;

(C5) $\frac{\epsilon_{0}}{2 H}\left(\frac{3}{4}-\frac{\bar{b}}{2}\right)>L_{h}$;

(C6) $\frac{b}{2}-\frac{2 H\left(L_{h}+1\right)}{\epsilon_{0}} \geq \frac{1}{4}$, where $\underline{b}=\min _{k}\left|b_{k}\right|$.

According to the result of [9], a bounded sequence $x_{i}$ is a solution of equation (1) if and only if the following relation is satisfied

$$
x_{i}=\sum_{j=-\infty}^{i} B^{i-j}\left(h\left(x_{j-1}\right)+\psi_{j-1}\right), i \in \mathbb{Z}
$$

Let us rewrite equation (2) in coordinate form:

$$
\begin{gathered}
x_{i}^{k}=\sum_{j=-\infty}^{i} b_{k}^{i-j}\left(h_{k}\left(x_{j-1}\right)+\psi_{j-i}^{k}\right), \\
i \in \mathbb{Z}, k=1, \ldots, p .
\end{gathered}
$$

The sequence $\varphi \in U, \varphi=\left\{\varphi_{i}\right\}, \varphi_{i}=$ $\left(\varphi_{i}^{1}, \varphi_{i}^{2}, \ldots, \varphi_{i}^{p}\right)$. Define on $U$ the operator $\Pi$ such that $\Pi \varphi=\left(\Pi_{1} \varphi, \Pi_{2} \varphi, \ldots, \Pi_{p} \varphi\right)$, and $\Pi_{k} \varphi=$ $\left\{\left(\Pi_{k} \varphi\right)_{i}\right\}, 1 \leq k \leq p, i \in \mathbb{Z}$, where

$$
\left(\Pi_{k} \varphi\right)_{i}=\sum_{j=-\infty}^{i} b_{k}^{i-j}\left(h_{k}\left(\varphi_{j-1}\right)+\psi_{j-i}^{k}\right) .
$$

Fix a sequence $\varphi \in U$. Then one can find that

$$
\begin{aligned}
\left|\left(\Pi_{k} \varphi\right)_{i}\right| & \leq \sum_{j=-\infty}^{i}\left|b_{k}^{i-j}\right|\left(M_{h}+M_{\psi}\right) \leq \\
& \leq\left(M_{h}+M_{\psi}\right) \frac{1}{1-\bar{b}},
\end{aligned}
$$

for $1 \leq k \leq p, i \in \mathbb{Z}$. Thus, by condition (C4) it implies that $\Pi \varphi \in U$ and condition (A1) is satisfied.

Let us fix arbitrary positive number $\varepsilon$ and an interval of integers $[\alpha, \beta]$. There exists an integer $\gamma<\alpha$ and a number $\xi>0$, which satisfy the following inequalities,

and

$$
\xi\left(L_{h}+1\right) \frac{1}{1-\bar{b}}<\frac{\varepsilon}{2}
$$

$$
2\left(M_{h}+M_{\psi}\right) \frac{\bar{b}^{\alpha-\gamma}}{1-\bar{b}}<\frac{\epsilon}{2} .
$$

There exists sufficiently large $n$ such that ||$\varphi_{i+\zeta_{n}-1}-\varphi_{i-1}||<\xi$ and $\left\|\psi_{i+\zeta_{n-1}}-\psi_{i-1}\right\|<$ $\xi$ for $i \in[\gamma, \beta]$. Then for all $i \in[\alpha, \beta]$ we have that

$$
\begin{gathered}
\left|\left(\Pi_{k} \varphi\right)_{i+\zeta_{n}}-\left(\Pi_{k} \varphi\right)_{i}\right|= \\
=\mid \sum_{j=-\infty}^{\gamma} b_{k}^{i-j}\left(h_{k}\left(\varphi_{j+\zeta_{n}-1}\right)-h_{k}\left(\varphi_{j-1}\right)+\right. \\
\left.+\psi_{j+\zeta_{n-1}}^{k}-\psi_{j-1}^{k}\right)+\sum_{j=\gamma+1}^{i} b_{k}^{i-j}\left(h_{k}\left(\varphi_{j+\zeta_{n-1}}\right)-\right. \\
\left.-h_{k}\left(\varphi_{j-1}\right)+\psi_{j+\zeta_{n}-1}^{k}-\psi_{j-1}^{k}\right) \mid \leq 2\left(M_{h}+\right. \\
\left.+M_{\psi}\right) \frac{\bar{b}^{\alpha-\gamma}}{1-\bar{b}}+\xi\left(L_{h}+1\right) \frac{1}{1-\bar{b}} .
\end{gathered}
$$

Thus, by inequalities (5) and (6), for large enough $n$ it is true that $\left|\left(\Pi_{k} \varphi\right)_{i+\zeta_{n}}-\left(\Pi_{k} \varphi\right)_{i}\right|<\varepsilon$ for all $1 \leq k \leq p$ and $i \in[\alpha, \beta]$. Since $\varepsilon$ is arbitrary small number the condition (A2) is valid.

For two sequences $a, b \in U$ the inequality

$$
\begin{aligned}
& \left|\left(\Pi_{k} a\right)_{i}-\left(\Pi_{k} b\right)_{i}\right|= \\
& =\left|\sum_{j=-\infty}^{i} b_{k}^{i-j}\left(h_{k}\left(a_{j}\right)-h_{k}\left(b_{j}\right)\right)\right| \leq \\
& \leq \frac{L_{h}}{1-\bar{b}}\|a-b\|_{1}
\end{aligned}
$$


is valid for all $i \in \mathbb{Z}$. Thus, we can conclude that $\|\Pi a-\Pi b\|_{1} \leq \frac{L_{h}}{1-\bar{b}}\|a-b\|_{1}$ for all $i \in \mathbb{Z}$, and by condition (C3) the operator $\Pi$ is contractive.

Theorem 1. Suppose that conditions (C1)-(C5) are valid, then the system (1) possesses unique asymptotically stable strongly unpredictable solution.

Proof. By contraction mapping theorem there exists the fixed point $\omega \in U$ of the operator $\Pi$ which is a bounded solution of the system (1) and it satisfies the inequality $\|\omega\|_{1}<H$.

Now, we prove the unpredictability of the solution $\omega=\left\{\omega_{i}\right\}, \omega_{i}=\left(\omega_{i}^{1}, \omega_{i}^{2}, \ldots, \omega_{i}^{p}\right)$ of the system (1). The coordinates of the sequence $\omega$ satisfy the relation (1), that is

$$
\begin{gathered}
\omega_{i+1}^{k}=b_{k} \omega_{i}^{k}+h_{k}\left(\omega_{i}\right)+\psi_{i}^{k}, \\
i \in \mathbb{Z}, k=1,2, \ldots, p .
\end{gathered}
$$

Fix a natural number $k=1,2, \ldots, p$, and $n \in \mathbb{N}$. Consider two alternatives, (i) $\left|\omega_{\zeta_{n}+\eta_{n}}^{k}-\omega_{\eta_{n}}^{k}\right|<\frac{\varepsilon_{0}}{2}$ and (ii) $\left|\omega_{\zeta_{n}+\eta_{n}}^{k}-\omega_{\eta_{n}}^{k}\right| \geq \frac{\varepsilon_{0}}{2}$.

(i) Using the relation

$$
\begin{gathered}
\omega_{\zeta_{n}+\eta_{n}+1}^{k}-\omega_{\eta_{n}+1}^{k}= \\
=b_{k}\left(\omega_{\zeta_{n}+\eta_{n}}^{k}-\omega_{\eta_{n}}^{k}\right)+h_{k}\left(\omega_{\zeta_{n}+\eta_{n}}\right)- \\
-h_{k}\left(\omega_{\eta_{n}}\right)+\psi_{\zeta_{n}+\eta_{n}}^{k}-\psi_{\eta_{n}}^{k}
\end{gathered}
$$

and condition (C5), we obtain for $n \in \mathbb{N}$ that

$$
\begin{gathered}
\left|\omega_{\zeta_{n}+\eta_{n}+1}^{k}-\omega_{\eta_{n}+1}^{k}\right| \geq \\
\geq\left|\psi_{\zeta_{n}+\eta_{n}}^{k}-\psi_{\eta_{n}}^{k}\right|-\left|b_{k}\left(\omega_{\zeta_{n}+\eta_{n}}^{k}-\omega_{\eta_{n}}^{k}\right)\right|- \\
-\left|h_{k}\left(\omega_{\zeta_{n}+\eta_{n}}\right)-h_{k}\left(\omega_{\eta_{n}}\right)\right| \geq \epsilon_{0}- \\
-\bar{b} \frac{\varepsilon_{0}}{2}-2 L_{h} H>\varepsilon_{0}- \\
-\bar{b} \frac{\varepsilon_{0}}{2}-\frac{\varepsilon_{0}}{2 H}\left(\frac{3}{4}-\frac{\bar{b}}{2}\right) 2 H \geq \frac{\varepsilon_{0}}{4} .
\end{gathered}
$$

(ii) For the case $\left|\omega_{\zeta_{n}+\eta_{n}}^{k}-\omega_{\eta_{n}}^{k}\right| \geq \frac{\varepsilon_{0}}{2}$, by relation (8) and condition (C6) we have that

$$
\begin{gathered}
\left|\omega_{\zeta_{n}+\eta_{n}+1}^{k}-\omega_{\eta_{n}+1}^{k}\right| \geq \underline{b} \frac{\varepsilon_{0}}{2}-2 L_{h} H-2 H \geq \\
\geq \varepsilon_{0}\left(\frac{\underline{b}}{2}-\frac{2 H\left(L_{h}+1\right)}{\varepsilon_{0}}\right) \geq \frac{\varepsilon_{0}}{4} .
\end{gathered}
$$

Thus, we obtained that $\left|\omega_{\zeta_{n}+\eta_{n}+1}^{k}-\omega_{\eta_{n}+1}^{k}\right| \geq$ $\frac{\varepsilon_{0}}{4}$. That the solution $\omega$ of system (1) is strongly unpredictable with positive number $\frac{\varepsilon_{0}}{4}$ and sequences $\zeta_{n}$ and $\eta_{n}+1$.

Using condition (C3) and the inequality (7), it is easy to verify that the solution $\omega$ of the system (1) is asymptotically stable [10]. The theorem is proved.

\section{An example}

Let us take into account the logistic discrete equation

$$
\lambda_{i+1}=\mu \lambda_{i}\left(1-\lambda_{i}\right) .
$$

The interval $[0,1]$ is invariant under the iterations of (9) for $\mu \in(0,4]$ [11]. In paper [1] was proved that the equation (9) has an unpredictable solution. Let us denote by $\rho_{i}, i \in \mathbb{Z}$, the unpredictable solution of the logistic map (9) with $\mu=3.91$. In this section we use the sequence $\rho_{i}$ as a perturbation.

Consider the system

$$
\begin{aligned}
& x_{i+1}=-\frac{2}{3} x_{i}+k \operatorname{arctg}\left(y_{i}\right)+l \rho_{i} \\
& y_{i+1}=\frac{3}{5} y_{i}+m \operatorname{arctg}\left(x_{i}\right)+n \rho_{i} .
\end{aligned}
$$

To satisfy conditions (C1)-(C6) we need to take $k, l, m, n$ quite small. To this end, we have chosen $k=\frac{1}{9}, l=\frac{3}{8}, m=\frac{1}{12}, n=-\frac{5}{11}$ and got the strongly unpredictable solution. Figures 1 and 2 represent the strongly unpredictable solution of the system (10) with initial data $\rho_{0}=0.35, x_{0}=0.25$ and $y_{0}=$ -0.95 . 


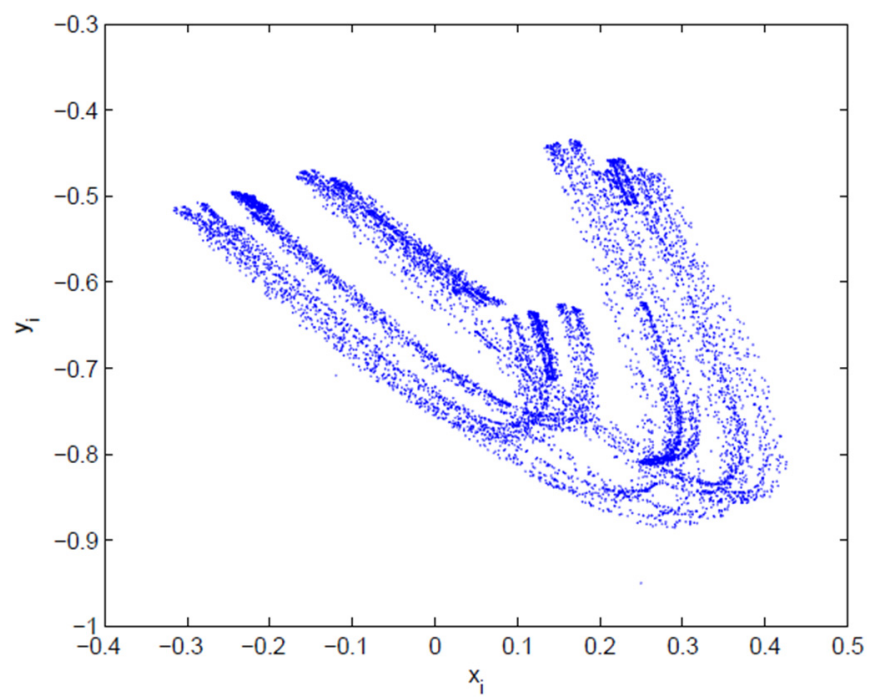

Figure 1 - The trajectory of system (10).

The figure manifests that the dynamics of system (10) is chaotic
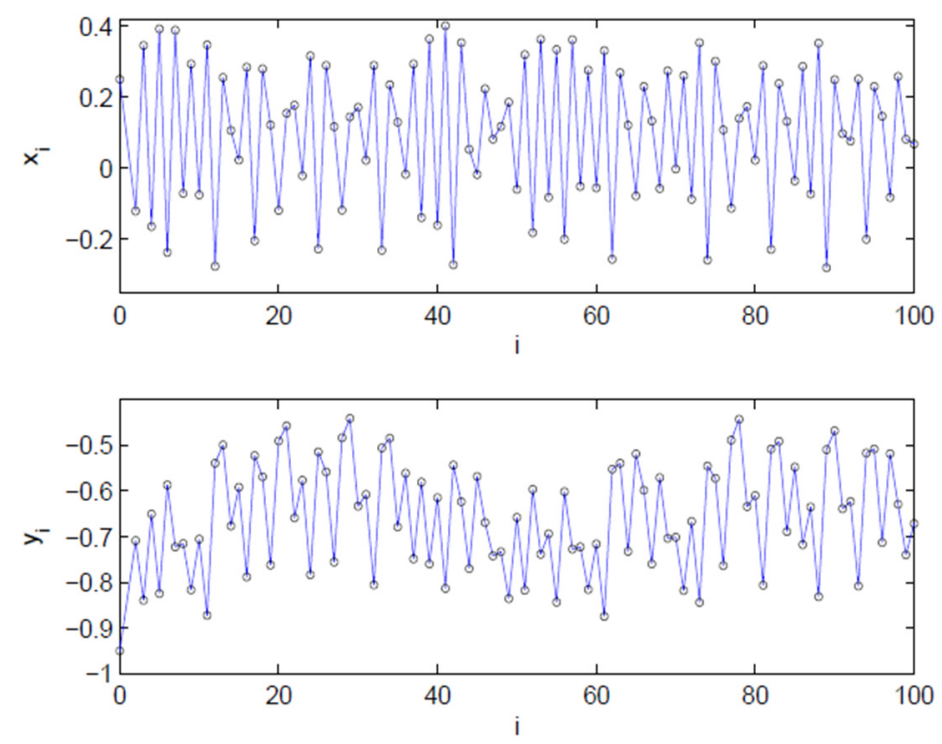

Figure 2 - The solution of system (10)

with the initial data $\rho_{0}=0.35, x_{0}=0.58$, and $y_{0}=-3.95$.

\section{Acknowledgements}

M. Akhmet has been supported by a grant (118F161) from TUBITAK, the Scientific and Technological Research Council of Turkey.

M. Tleubergenova and A. Zhamanshin have been supported by the Ministry of Education and Science of the Republic of Kazakhstan under grant No. (AP05132573) "Cellular neural networks with continuous/discrete time and singular perturbations" (2018-2020).

\section{References}

1. Akhmet M.U., Fen M.O., "Poincare chaos and unpredictable functions", Communications in Nonlinear Science and Numerical Simulation, vol. 48 (2017): 85-94. 
2. Akhmet M. and Fen M.O., "Unpredictable points and chaos", Communications in Nonlinear Science and Numerical Simulation, (2016).

3. Akhmet M., Seilova R., Tleubergenova M., Zhamanshin A., SICNNs with strongly unpredictable oscillations, IEEE Transactions on Neural Networks and Learning Systems, submitted (2019).

4. Aihara T., Toyoda M., Chaotic neural networks, Physics Letters A, vol. 144 No. 6-7 (1990): 333-340.

5. Field, R.J., Györgyi, L.: Chaos in Chemistry and Biochemistry, World Scientific, Singapore (1993)

6. Gonzales-Miranda, J.M.: Synchronization and Control of Chaos, Imperial College Press, London (2004)
7. Dauylbaev M. K., "The asymptotic behavior of solutions to singularly perturbed nonlinear integro-differential equations", Siberian Mathematical Journal, vol. 41, No. 1 (2000): 49-60.

8. Akhmet M., Dauylbayev M., Mirzakulova A., A singularly perturbed differential equation with piecewise constant argument of generalized type, Turkish Journal of Mathematics. vol. 42. No. 4. (2018): 1680 - 1685.

9. Lakshmikantham V., Trigiante D., Theory of difference equations: numerical methods and applications, USA, Marcel Dekker, (2002).

10. Agarwal P., Difference Equations and Inequalities, Marcel Dekker, (1992).

11. Hale J., Kocak H., Dynamics and bifurcations, New York, Springer-Verlag, (1991). 